Applications of Photonuclear Physics for International Safeguards and Security

M. S. Johnson, J. M. Hall, D. P. McNabb, J. McFarland, E. Norman, W. Bertozzi, S. Korbly, R. Ledoux, W. Park

April 21, 2010

Nuclear Data 2010

Jeju Island, South Korea

April 26, 2010 through April 30, 2010 
This document was prepared as an account of work sponsored by an agency of the United States government. Neither the United States government nor Lawrence Livermore National Security, LLC, nor any of their employees makes any warranty, expressed or implied, or assumes any legal liability or responsibility for the accuracy, completeness, or usefulness of any information, apparatus, product, or process disclosed, or represents that its use would not infringe privately owned rights. Reference herein to any specific commercial product, process, or service by trade name, trademark, manufacturer, or otherwise does not necessarily constitute or imply its endorsement, recommendation, or favoring by the United States government or Lawrence Livermore National Security, LLC. The views and opinions of authors expressed herein do not necessarily state or reflect those of the United States government or Lawrence Livermore National Security, LLC, and shall not be used for advertising or product endorsement purposes. 


\title{
APPLICATIONS OF PHOTONUCLEAR PHYSICS FOR INTERNATIONAL SAFEGUARDS AND SECURITY
}

\author{
M. S. Johnson*, J. M. Hall, D. P. McNabb, J. L. McFarland ${ }^{1}$, E. B. Norman ${ }^{1}$, W. Bertozzi² ${ }^{2}$, S.E. Korbly ${ }^{2}$, R.J. Ledoux², and \\ W.H. Park ${ }^{2}$ \\ Lawrence Livermore National Laboratory, Livermore, CA 94550 \\ ${ }^{1}$ University of California, Berkeley, CA 94720 \\ ${ }^{2}$ Passport Systems Incorported, Acton, MA 01720 \\ ${ }^{*}$ Corresponding author. E-mail : johnson329@1lnl.gov \\ Received \\ Accepted for Publication
}

Studies of nuclear resonance fluorescence based applications are presented. Important for these applications are data for isotopes such as ${ }^{239} \mathrm{Pu}$. Nuclear resonance fluorescence measurements of ${ }^{239} \mathrm{Pu}$ were performed at the free electron laser facility at UC Santa Barbara using photons from a bremsstrahlung beam with an endpoint energies between $4.0 \mathrm{MeV}$ and 5.5 MeV. Though no discrete states with significant confidence level were measured, we have excluded the region above 27(3) eV-barns, or 4-sigma, where we would expect only a small chance of false positives. Details of the measurements and the results are presented here.

KEYWORDS : ND2010, Nuclear Data, NRF, Nuclear resonance fluorescence

\section{INTRODUCTION}

High-precision nuclear data is critical to application programs based on nuclear reactions. Many global security and international safeguards programs are studying and developing systems to identify and characterize nuclear and radiological materials. Two classes of systems that cover different areas of these applications are passive and active technologies. Passive technologies rely on the emitted radiation of unstable nuclei. Passive systems are desirable but the current technology lacks certain features that could cover a broad application space. For example, gamma-ray tracking [1] is not yet developed so that passive imaging could be useful to map configurations of radiological materials. Passive detection relies on unstable nuclei but isotopic information about non-radioactive material could be useful. This leads to the second class of technologies, active interogation. One simple example is radiography, which is used in current security applications, medical applications, industrial aplications, etc... These systems utilize external photon sources to stimulate the material and measure the resulting signals to infer the material composition. One type of active interrogation with photon sources that is at the forefront of applied science for security applications is a technology that uses nuclear resonance fluorescence (NRF) [2,3]. NRF provides a unique approach to study nuclear isotopes. NRF is a process in which a photon, with energy equivalent to a resonance level of a particular nucleus, excites the nucleus to the resonant level. The nucleus then decays to the ground state by a direct transition or multi-step transitions through intermediate levels. The efficacy of NRF-based detection, imaging and assay systems depend on knowledge of NRF states of the isotope being probed.

As a research tool, NRF has been used to probe collective excitations in deformed rare-earth and actinide nuclei (Refs. [4-6] and references therein and more recently by some of us [7] and others [8,9]). This paper describes recent measurements of NRF excitations ${ }^{239} \mathrm{Pu}$ between 2.5 - and 3.8-MeV. We also look at lower levels previously discovered in ${ }^{239} \mathrm{Pu}$ [7]. The next section discusses some of the NRFbased applications being studied at Lawrence Livermore National Laboratory (LLNL).

\subsection{Applications}

NRF is being studied at LLNL for a variety of securityand safeguards-based applications. For example, we have studied detection protocols to identify heavily shielded materials (c.f. [11]). We have developed an analytical model [11] to predict count rates to detect heavily shielded materials. Important to the model are the nuclear data regarding the NRF strengths and backgrounds (e.g. [10]). The results from our experiments at HIGS (High-Intensity Gamm-ray Source) demonstrated that it is possible to extract spatially integrated information on the isotopic composition of an object, such as a spent fuel rod for nuclear safeguards applications, from NRF measurements. The potential use of tightly collimated NRF probe beams to take radiographic and/or tomographic images of an object, thereby allowing one to generate spatially resolved maps of isotopic 
Simulated 2 MeV e-Brem X-Ray CT image
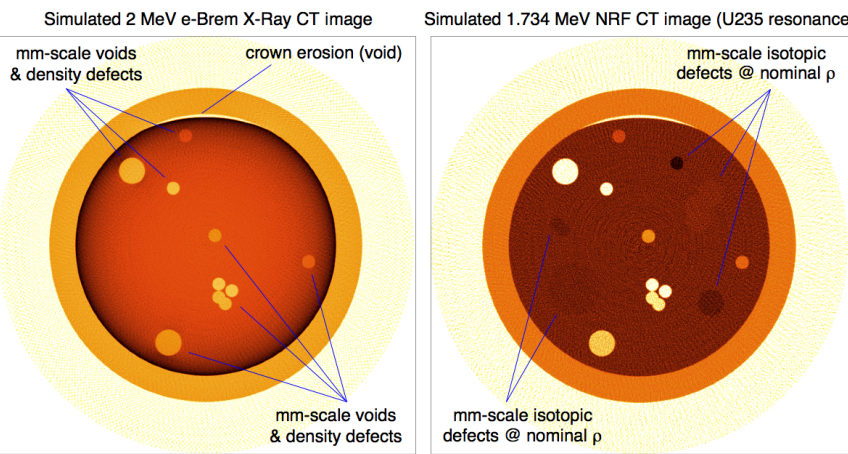

Fig. 1. Comparison of simulations of bremsstrahlung-based CT (left) and quasi-monoenergetic-based CT (right) images at 1.7 MeV. The simulations were based on a fixed counting time. See text for more details.

composition, has also been explored using detailed Monte Carlo simulations. Figure 1 shows slices through simulated $\mathrm{CT}$ reconstructions of a conventional $\mathrm{Zr}$-clad $\mathrm{UO}_{2}$ fuel element imaged using a standard $2 \mathrm{MeV}$ e-Bremsstrahlung source (Fig. 1, left) and an NRF source tuned to the 1.734 MeV NRF resonance in ${ }^{235} \mathrm{U}$ (Fig. 1, right). Each CT scan consisted of 640 sequential measurements spanning a range of $1.60 \mathrm{~cm}$ (spatial resolution $25 \mu \mathrm{m}$ ) at each of 149 different viewing angles. The simulated defects in the $\mathrm{UO}_{2}$ fuel are $0.50 \mathrm{~mm}, 1.00 \mathrm{~mm}$ and $2.00 \mathrm{~mm}$ in diameter. The mass density defects range from $10 \%$ to $80 \%$ of nominal $\left(10.96 \mathrm{~g} / \mathrm{cm}^{3}\right)$ and the isotopic defects range from $0.20 \%$ to $25.0 \%$ (nominal is assumed to be $3 \%$ ). These simulations indicate that NRF imaging systems could potentially identify and map not only sub-mm-scale structural and density defects in fuel elements, but also isotopic defects and/or trace actinide concentrations.

In a real safeguards scenario the amount of plutonium in a fuel rod may be important (versus ${ }^{235} \mathrm{U}$ for the example above). We have previously measured some lower-lying states ${ }^{239} \mathrm{Pu}$ [7]. However, for penetrability higher-lying resonances are important. Also, finding stronger resonances would benefit safeguards applications, especially those that study full fuel assemblies. We have made measurements looking for higher-lying and stronger resonances in ${ }^{239} \mathrm{Pu}$. The details of those measurements are given below.

\section{EXPERIMENTAL METHOD}

Measurement probes for NRF states in ${ }^{239} \mathrm{Pu}$ were conducted using the $\left(\gamma, \gamma^{\prime}\right)$ reaction with bremsstrahlung photons. The experimental setup was located at The Center for Terahertz Science and Technology at the University of California at Santa Barbara. The photons were produced by accelerating electrons with a $6 \mathrm{MV}$ pelletron and impinging the electrons on a "radiator" of 3-mm thickness of water cooled copper. A disk of ${ }^{27} \mathrm{Al}$ was placed in front of the $\mathrm{Pu}$ target to normalize any observed NRF peaks associated with the $\mathrm{Pu}$ material and to determine the flux of the beam.
Measurements of ${ }^{239} \mathrm{Pu}$ used end-point energies between 4.0- and 5.5-MeV to probe for NRF strengths based on optimization of signal strength to background. The bremsstrahlung photons pass through a collimator with a $2.5^{\circ}$ half angle, conical opening. Additional shielding was placed around the radiator to reduce background. The NRF targets were placed $100 \mathrm{~cm}$ from the radiator and the entire target and holder was completely illuminated by the bremsstrahlung photons.

The $\mathrm{Pu}$ target contained approximately 4 grams of $\sim 93 \%{ }^{239} \mathrm{Pu}$. Each $\mathrm{Pu}$ target was $1.5 \mathrm{~cm}$ in diameter, $1.5 \mathrm{~mm}$ thick, and was encapsulated in 25 grams of Nitronic-40 with a 1-mil stainless steel window. The manganese of the Nitronic-40 (and other elements) did produce extraneous photopeaks, which served as an energy calibration. The outer diameter of the Nitronic-40 holder was approximately $2.5 \mathrm{~cm}$. For normalization, thin disks of $\mathrm{Al}$ (approximately $1.2 \mathrm{~mm}$ thick and $1.6 \mathrm{~cm}$ in diameter) were placed directly in front of the Pu target.

The $\gamma$-rays were measured with a cluster of $6 \mathrm{HPGe}$ detectors at 120-degrees with respect to the beam direction in the lab frame. All detectors were encased in thick $\mathrm{Pb}$ shielding to reduce background from room scatter. The openings to the detectors were covered with $\mathrm{Pb}$ absorbers of thickness $3 / 4$ " to reduce low-energy triggers.

\section{RESULTS}

The data for various endpoint energies were collected and analyzed for known and unknown peaks and structures. Although no new peaks associated with NRF states associated with the $\mathrm{Pu}$ target were observed, we were able to set an upper limit to the integrated cross-sections expected with varying confidence levels. The region of maximum sensitivity for observing NRF scattering with a 4.0 MeV endpoint energy bremsstrahlung source is between $2.4-$ and $3.8-\mathrm{MeV}$. For simplicity we focus on the data set with $4.0 \mathrm{MeV}$ endpoint energy for the remainder of the paper. The region of sensitivity was determined by estimating, in the given acquisition time, the confidence level of measurable peaks. In simpler terms, for energies above the endpoint energy there are no source photons from the bremsstrahlung source. And below $2.4 \mathrm{MeV}$, the large backgrounds rapidly reduce the sensitivity. The dominant backgrounds below $2.4 \mathrm{MeV}$ are associated with Compton back-scattering of the beam and Compton scattering from the natural ubiquitous background radiation line at $2.615 \mathrm{MeV}$. The detector integrated spectra for the $\mathrm{Pu}$ target and holder versus the holder only is displayed in figure 2.

The data collected, for all endpoint energies, had a high-energy background with a shape that is consistent with Compton scattering from neutron capture and inelastic processes on light nuclei (See Fig. 2). The data indicated that the Compton rate from neutron processes was not sensitive to the end point energy. It was determined that the source of neutrons resulting in this high-energy background was from the cooling water in the bremsstrahlung converter. 


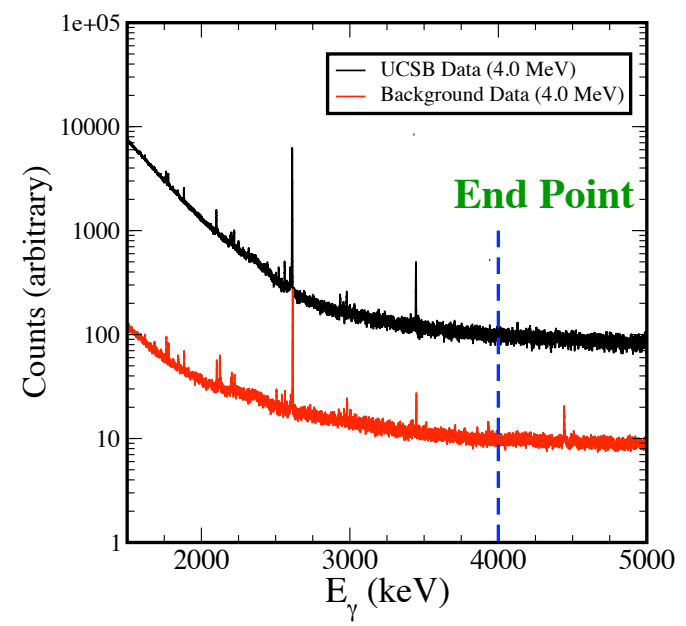

Fig. 2. Comparison of UCSB data run (black) and background (red) run at $4.0 \mathrm{MeV}$. The flat high-energy continuum is Compton background form high-energy gamma rays from neutron processes on light nuclei. See text for more details.

Bremsstrahung photons and residual electrons were causing disintegration of the natural abundance of deuterium in the water leading to neutron production. This background wasn't observed in our previous measurements [7]. This was because the cooling jacket for that converter was not in the beam path. Monte Carlo simulations for this setup were performed [12] and validate the neutron production rate and subsequently the flat background.

Clearly visible in figure 2 are peaks in both the $\mathrm{Pu}$ spectrum and background spectrum. Upon closer analysis of our region of sensitivity, between 2.4- and 3.8-MeV, it was determined that the peaks are the same in both spectra. More specifically, these peaks are equivalent in centroid and in strength. Also, all of the peaks have either been identified as NRF peaks from the target holder or experimental apparatus or associated with neutron processes. A search for low confidence level peaks was performed but no peaks associated with the Pu target could be determined. That is not to say that no NRF states exist between 2.4- and 3.8$\mathrm{MeV}$. We have performed an estimate to determine at what level we can exclude certain NRF strengths.

We have identified and listed many of the dominant peaks seen in Fig. 2 in table 1 (See Ref. [12] for more detailed explanation). A sample of boron was included in the background runs. Since the endpoint energy for the data in Fig. 2 and in Tab. 1 is $4.0 \mathrm{MeV}$ any presence of higher energy transitions in ${ }^{11} \mathrm{~B}$ can only be caused by the $(\mathrm{n}, \gamma)$ reaction. Indeed, the data shows (See Tab. 1) that neutrons are present in the background. Further comparison of the beam-on and beam-off runs show that the boron activation lines are only present when the beam is on, validating the source of neutrons as beam related.

The estimates were made using the ratio method discussed in [7] (and references therein). We incorporated the attenuation of the flux going into the midpoint of the target and the attenuation of the signal from the target
Table 1. Descriptions of the peaks identified in Fig. 2. All peaks that appear below were discovered in both the Pu data and the background data. The exception are the boron peaks ${ }^{\dagger}$ which only appear in the background data. The $5019 \mathrm{keV}$ line for boron" is not shown in Fig. 2 but included here and discussed in the text.

The $1779-\mathrm{keV}$ transition* and $1811-\mathrm{keV}$ transition** may be inelastic neutron scattering on ${ }^{28} \mathrm{Si}$ and ${ }^{56} \mathrm{Fe}$, repectively. Excluded in this table are natural backgrounds and escape peaks.

\begin{tabular}{ccc}
\hline Isotope & Transition Energy $(\mathrm{keV})$ & Reaction Type \\
\hline${ }^{239} \mathrm{Pu}$ & 2143 & $\left(\gamma, \gamma^{\prime}\right)$ \\
${ }^{239} \mathrm{Pu}$ & 2423 & $\left(\gamma, \gamma^{\prime}\right)$ \\
${ }^{27} \mathrm{Al}$ & 2212 & $\left(\gamma, \gamma^{\prime}\right)$ \\
${ }^{27} \mathrm{Al}$ & 2982 & $\left(\gamma, \gamma^{\prime}\right)$ \\
${ }^{27} \mathrm{Al}$ & 3004 & $\left(\gamma, \gamma^{\prime}\right)$ \\
${ }^{27} \mathrm{Al}$ & 3034 & $(\mathrm{n}, \gamma)$ \\
${ }^{27} \mathrm{Al}$ & 3465 & $(\mathrm{n}, \gamma)$ \\
${ }^{28} \mathrm{Al} \mathrm{l}^{*}$ & 1779 & $\beta$-delayed $\gamma$ \\
${ }^{56} \mathrm{Fe}$ & 2113 & $\left(\mathrm{n}, \mathrm{n}^{\prime} \gamma\right)$ \\
${ }^{56} \mathrm{Fe}$ & 2523 & $\left(\mathrm{n}, \mathrm{n}^{\prime} \gamma\right)$ \\
${ }^{56} \mathrm{Fe}$ & 2959 & $\left(\mathrm{n}, \mathrm{n}^{\prime} \gamma\right)$ \\
${ }^{56} \mathrm{Fe}$ & 3448 & $\left(\gamma, \gamma^{\prime}\right)$ \\
${ }^{10} \mathrm{~B}^{\dagger}$ & 2125 & $(\mathrm{n}, \gamma)$ \\
${ }^{10} \mathrm{~B}^{\dagger}$ & 2895 & $(\mathrm{n}, \gamma)$ \\
${ }^{10} \mathrm{~B} \dagger$ & 4444 & $(\mathrm{n}, \gamma)$ \\
${ }^{10} \mathrm{~B}^{\ddagger}$ & 5019 & $(\mathrm{n}, \gamma)$ \\
${ }^{55} \mathrm{Mn}$ & 2198 & $\left(\gamma, \gamma^{\prime}\right)$ \\
${ }^{55} \mathrm{Mn}$ & 2252 & $\left(\gamma, \gamma^{\prime}\right)$ \\
${ }^{55} \mathrm{Mn}$ & 2563 & $\left(\gamma, \gamma^{\prime}\right)$ \\
${ }^{55} \mathrm{Mn}$ & 2695 & $\left(\mathrm{n}, \mathrm{n}^{\prime} \gamma\right)$ \\
${ }^{55} \mathrm{Mn}$ & 2753 & $\left(\gamma, \gamma^{\prime}\right)$ \\
${ }^{55} \mathrm{Mn}$ & 3195 & $\left(\mathrm{n}, \mathrm{n}^{\prime} \gamma^{\prime}\right)$ \\
${ }^{56} \mathrm{Mn}{ }^{* *}$ & 1811 & $\beta$-delayed $\gamma$ \\
${ }^{1} \mathrm{H}$ & 2223 & $(\mathrm{n}, \gamma)$ \\
${ }^{52} \mathrm{Cr}$ & 2321 & $(\mathrm{n}, \gamma)$ \\
${ }^{53} \mathrm{Cr}$ & 2239 & $(\mathrm{n}, \gamma)$ \\
${ }^{14} \mathrm{~N}$ & 1885 & $(\mathrm{n}, \gamma)$ \\
${ }^{35} \mathrm{Cl}$ & 2447 & $(\mathrm{n}, \gamma)$ \\
\hline${ }^{55}$ & &
\end{tabular}

to the detector whilst including all intervening materials. The attenuation coefficients were obtained from Ref. [13]. Doppler broadening was included as well as self-absorption of the NRF signal. Energy dependence of the flux and detector efficiencies were included. The spectra were summed using gain correction for proper alignment. Dead time corrections were also applied. The Pu estimates were then normalized to the measured Al signal and plotted as a function of confidence level (Fig. 3). The lower extents of the contours shown in figure 3 indicate regions where the statistical significance is so low that peaks may not be measurable with a high degree of confidence. In other words, the figure 3 shows the exclusion areas of integrated cross-sections at varying confidence levels. There are two things that are clearly evident in Fig. 3, first, a marked increase in the measurable integrated cross-sections near 4.0 MeV. This is because $4.0 \mathrm{MeV}$ is the endpoint energy which implies there is a drop in gamma-ray flux. Second, the confidence levels start to increase below 2.4 MeV. This 
M. S. Johnson et al., Applications of Photonuclear Physics for International Safeguards and Security

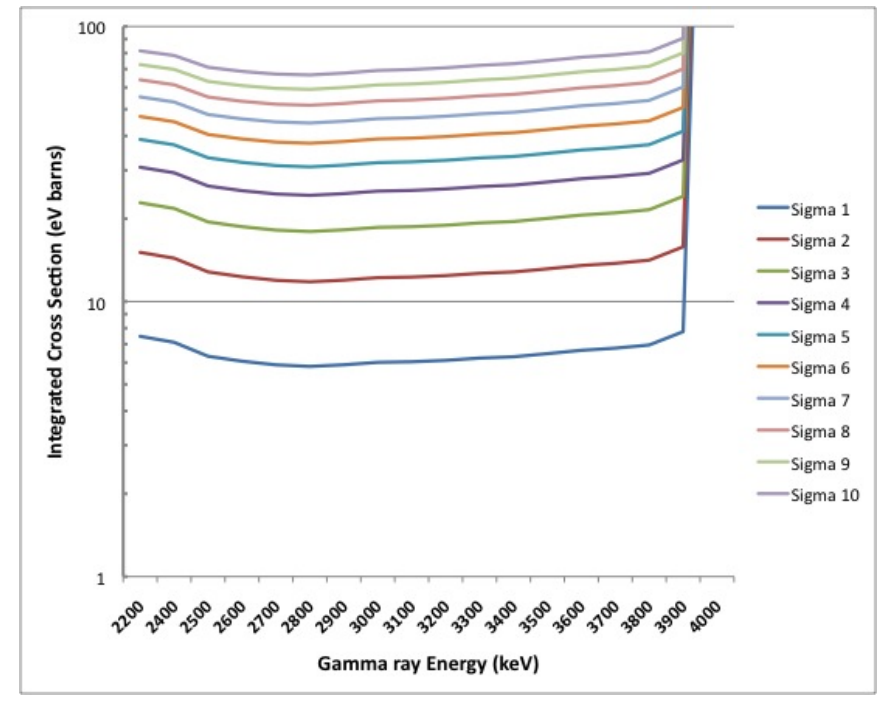

Fig. 3. Exclusion plot of NRF strengths with a $4.0 \mathrm{MeV}$ bremsstrahlung source. Each curve represents the integrated NRF cross-section one expects to see with a given confidence level.

The lower curves are lower confidence levels. The higher cross-sections are expected to have a higher signal-to-noise signature. The flatness over the region of sensitivity is due to flat backgrounds. The higher exclusion values $(\approx 6 \mathrm{eV}$ barns $)$ are caused by neutron backgrounds. The upturn below $\approx 2500 \mathrm{keV}$ is a result of the Compton edge of the naturally occurring $2615 \mathrm{keV}$ line. The sharp upturn at $4000 \mathrm{keV}$ is a result of the deficit of flux at the endpoint energy.

is caused by the Compton scatter of the ubiquitous naturally occurring background radiation line at $2.615 \mathrm{MeV}$.

We have compared this data to our previous measurements of ${ }^{239} \mathrm{Pu}$ [7]. The strongest states for ${ }^{239} \mathrm{Pu}$ are $2143 \mathrm{keV}$ and $2423 \mathrm{keV}$ (see Ref. [7]) and were measured to be $13(2) \mathrm{eV}$ barns and $10(2) \mathrm{eV}$ barns, respectively. If these peaks are in fact associated with ${ }^{239} \mathrm{Pu}$ then for the measurements here, we estimate the peaks should be measurable at the 1.5 sigma level. More specifically, at the 1.5-sigma level, peaks with integrated cross-sections of 11(4) eV barns and 10(2) eV barns should be measured in the vicinities of $2143 \mathrm{keV}$ and $2423 \mathrm{keV}$, respectively. For the data presented here, the peaks have been measured with areas and uncertainties, 53(43) and 53(57) (i.e. 1sigma) for $2143 \mathrm{keV}$ and $2423 \mathrm{keV}$, respectively. Given the uncertainties in the estimates we are confident that the estimates and the measured values are consistent. The variance between 1- and 1.5-sigma is most likely caused by our simplistic estimates.

From the data analysis described above and the exclusion plot in figure 3 we have excluded the region above 27(3) eV-barns, or 4-sigma, where we would expect only a small chance of a false positive ( $\lesssim 0.02$ occurrences). We expect that the presence of the valence neutron of ${ }^{239} \mathrm{Pu}$ to divide the strength of the dipole motion amongst the different spin projections on the symmetry axis. Therefore, the NRF states in this region would have low cross-sections as indicated by the exclusion analysis performed here and the results from our prior paper [7].

\section{CONCLUSION}

There are many possible applications for NRF. We are exploring different security and safeguards applications that can exploit NRF. NRF simulations are being developed to be used to model potential applications. Benchmarking measurements are being performed to validate models. NRF measurements are being performed to identify states that can be used.

Recently, NRF measurements were performed using photons from a bremsstrahlung beam with an endpoint energies between 4.0 MeV and 5.5 MeV. Though no discrete states with significant confidence level were measured, we were able to exclude the region above 27(3) eV-barns, or 4sigma. Measurements of this region need to be performed with higher intensity beams to find NRF peaks and/or to validate the results of this work.

Preferably, beams that are $100 \%$ polarized and quasimonoenergetic are useful at characterizing NRF states with respect to parity and to a certain extent, isotopic verification. Coincidence measurements are also important to look for branchings towards photofission and photodisintegration. These are particularly relevant in actinides such as $\mathrm{Pu}$ where the cross-sections for these processes are much larger than NRF at certain energies.

\section{ACKNOWLEDGMENTS}

LLNL's contributions were funded by the U.S. DOE/NNSA and DHS/DNDO. LLNL's work performed under the auspices of the U.S. DOE by LLNL under Contract DE-AC52-07NA27344. Passport supported in part by DHS/DNDO, SPAWAR contract N66001-05-D-6011/0002 and N66001-07-D-0025/0001. The UC Berkeley group was supported in part through an ARI grant from DHS/DNDO. Special thanks to UCSB FEL facility and staff.

\section{REFERENCES}

[1] I. Y. Lee, Nucl. Inst. E Meth. A422,195 (1999).

[2] W. Bertozzi and R. Ledoux, Nucl. Inst. $\mathcal{F}$ Meth. B241, 820 (2005).

[3] J. Pruet et al., J. Appl Phys. 99, 123102 (2006).

[4] J. Margraf et al., Phys. Rev. C. 42, 771 (1990).

[5] A. Zilges et al., Phys. Rev. C. 52, R468 (1995).

[6] H. H. Kneissl et al., Prog. Part. Nucl. Phys. 37, 349 (1996).

[7] W. Bertozzi et al., Phys. Rev. C. 78, 041601 (2008).

[8] S. Hammond et al., Phys. Rev. C. to be submitted (2010).

[9] E. Kwan et al., Phys. Rev. C. to be submitted (2010).

[10] C. A. Hagmann et al., J. Appl Phys., 106084901 (2009).

[11] M. S. Johnson et al., J. Appl Phys., to be submitted (2010).

[12] J. L. McFarland, Master's Thesis UC Berkeley (2009).

[13] M. J. Berger et al., XCOM: Photon Cross Section Database National Institute of Standards and Technology, NBSIR 873597 (1998). 